\title{
Improved Silbey-Harris polaron ansatz for the spin-boson model
}

\author{
Shu $\mathrm{He}^{1,2}$, Liwei Duan ${ }^{1}$, and Qing-Hu Chen ${ }^{1,3, *}$ \\ 1 Department of Physics, Zhejiang University, Hangzhou 310027, China \\ 2 Department of Physics and Electronic Engineering, \\ Sichuan Normal University, Chengdu 610066, China \\ 3 Collaborative Innovation Center of Advanced Microstructures, Nanjing 210093, China
}

(Dated: October 13, 2018)

\begin{abstract}
In this paper, the well-known Silbey-Harris (SH) polaron ansatz for the spin-boson model is improved by adding orthogonal displaced Fock states. The obtained results for the ground-state in all baths converge very quickly within finite displaced Fock states and corresponding SH results are corrected considerably. Especially for the sub-Ohmic spin-boson model, the converging results are obtained for $0<s<1 / 2$ in the fourth-order correction and very accurate critical coupling strengths of the quantum phase transition are achieved. Converging magnetization in the biased spin-boson model is also arrived at. Since the present improved SH ansatz can yield very accurate, even almost exact results, it should have wide applications and extensions in various spin-boson model and related fields.
\end{abstract}

PACS numbers: 03.65.Yz, 03.65.Ud, 71.27.+a, 71.38.k

\section{INTRODUCTION}

The celebrated spin-boson model [1, 2] describes a qubit (two-level system) coupled with a dissipative environment represented by a bath of continuous bosonic modes. There is currently considerable interest in this quantum many-body system due to the rich physics of quantum criticality and decoherence [2-4], applications to the emerging field of quantum computations [5], quantum devices [6], and quantum biology [7, 8]. It is widely used to study the microscopic behavior of open quantum systems [1]. The coupling between the qubit and the environment is characterized by a spectral function $J(\omega)$ which is proportional to $\omega^{s}$. The spectral exponent $s$ varies the coupling into three different cases: sub-Ohmic $(s<1)$, Ohmic $(s=1)$, and super-Ohmic $(s>1)$.

As a paradigmatic model to study the influence of environment on the quantum system, the spin-boson model has been extensively and persistently studied by many analytical and numerical approaches. On the analytical side, a pioneer work is undoubtedly the variational study based on the polaronic unitary transformation also known as Silbey-Harris ansatz (SH) ansatz [9]. A similar analytical approach was developed to study both static and dynamical behavior of the dissipative two-level system [10]. The original symmetric SH ansatz using a single coherent state was generalized to the asymmetric form for the sub-Ohmic baths [11]. Recently, the asymmetric SH ansatz was modified by superposing more than one nonorthogonal coherent states on the equal footing 12 14], and the equilibrium reduced density matrix in the SH frames was corrected to the second-order [15, 16].

On the numerical side, almost all advanced numerical approaches in the quantum many-particle physics have been applied and extended to this model and many interesting results have been obtained. The numerical renormalization group was applied at the earlier stage [17 for the sub-Ohmic baths, yielding non-mean-field critical exponents of the quantum phase transitions (QPT) for $0<s<1 / 2$ due to the Hilbert-space truncation error and the mass flow error 18 20]. Later on, quantum Monte Carlo (QMC) simulations based on an imaginary time path integral 21], sparse polynomial space approach [22], and exact diagonalization in terms of shift bosons [23] have sequentially developed and all found the mean-field critical exponent for $0<s<1 / 2$. The density matrix renormalization group was also applied, but not successful in the analysis of the critical phenomena 24]. More recently, using the density matrix renormalization group algorithm combined with the optimized phonon basis, a variational matrix product state approach formulated on a Wilson chain [25] was developed and the Hilbert-space truncation can be alleviated systematically. An alternative to the conventional matrix product state representation was also proposed [26]. Most recently, a highly efficient numerical method based on a time-dependent variational principle for matrix product states [27-29] was proposed and extended to simulate quantum dynamics of the spin-boson model.

Analytical exact study should be very challenging in the spin-boson model due to the infinite modes of the baths, unlike the single mode case [30-32]. Even the well controlled and fast converging analytical study was still lacking until now. In this paper, we propose an analytical approach for the ground state (GS) of the spin-boson model anchored to SH polaron ansatz. The bosonic state is expanded in the orthogonal displaced Fock states basis so that the $\mathrm{SH}$ results can be improved systematically. The high order displaced Fock states can essentially include the many-body correlations for bosons, which are obviously lacking in the SH wavefunction, so the results with increasing orders should approach the exact ones asymptotically.

The paper is organized as follows. In Sec. II, we briefly introduce the spin-boson model, and describe our improved SH ansatz in terms of displaced Fock states in 
detail. In addition, we test the approach in the single mode case. In Sec. III, we apply it to the spin-boson model for different baths, and further extend it to the biased spin-boson model. A short summary is given in Sec.IV.

\section{MODEL AND DISPLACED FOCK STATES}

The Hamiltonian of the spin-boson model is given by

$$
H=-\frac{\Delta}{2} \sigma_{x}+\sum_{k} \omega_{k} a_{k}^{\dagger} a_{k}+\frac{1}{2} \sigma_{z} \sum_{k} g_{k}\left(a_{k}^{\dagger}+a_{k}\right),
$$

where $\sigma_{x}$ and $\sigma_{z}$ are Pauli matrices, $\Delta$ is the tunneling amplitude between two levels, $\omega_{k}$ and $a_{k}^{\dagger}$ are the frequency and creation operator of the $k$-th harmonic oscillator, and $g_{k}$ is the interaction strength between the $k$-th bosonic mode and the local spin. The spin-boson coupling is characterized by the spectral function,

$$
J(\omega)=\pi \sum_{k} g_{k}^{2} \delta\left(\omega_{k}-\omega\right)=2 \pi \lambda \omega_{c}^{1-s} \omega^{s}, 0<\omega<\omega_{c}
$$

with $\omega_{c}$ a cutoff frequency. The dimensionless parameter $\lambda$ denotes the coupling strength.

To outline the approach more intuitively, we first consider the case without symmetry breaking. By using $|\uparrow\rangle$ and $|\downarrow\rangle$ to represent the eigenstate of $\sigma_{z}$, the GS wavefunction can be in principle expressed in the following standard set of complete orthogonal basis $\prod_{i=0}^{n} a_{k_{i}}^{\dagger}|0\rangle$ in Fock space

$$
\begin{aligned}
& \left|\Psi^{\prime}\right\rangle=\left(1+\sum_{k} \alpha_{k} a_{k}^{\dagger}+\sum_{k_{1}, k_{2}} u_{k_{1}, k_{2}} a_{k_{1}}^{\dagger} a_{k_{2}}^{\dagger}+\ldots\right)|0\rangle|\uparrow\rangle \\
& +\left(1-\sum_{k} \alpha_{k} a_{k}^{\dagger}+\sum_{k_{1}, k_{2}} u_{k_{1}, k_{2}} a_{k_{1}}^{\dagger} a_{k_{2}}^{\dagger}+\ldots\right)|0\rangle|\downarrow\rangle, \quad(3)
\end{aligned}
$$

where $|0\rangle$ is vacuum of bath modes, $\alpha_{k}, u_{k_{1} k_{2}}, \ldots$ are the coefficients, and even parity is considered. However, it is impossible to get reasonable results by performing direct diagonalization in this Fock space because of the huge Hilbert-space. Alternatively, the wavefunction (3) can be expressed in terms of another set of complete orthogonal basis, $D\left(\alpha_{k}\right) \prod a_{k_{i}}^{\dagger}|0\rangle$, where $D\left(\alpha_{k}\right)=$ $\exp \left[\sum_{k} \alpha_{k}\left(a_{k}^{\dagger}-a_{k}\right)\right]$ is an unitary operator, as

$$
\begin{aligned}
& |\Psi\rangle=D\left(\alpha_{k}\right)\left(1+\sum_{k_{1}, k_{2}} b_{k_{1} k_{2}} a_{k_{1}}^{\dagger} a_{k_{2}}^{\dagger}+\ldots\right)|0\rangle|\uparrow\rangle \\
& +D\left(-\alpha_{k}\right)\left(1+\sum_{k_{1}, k_{2}} b_{k_{1} k_{2}} a_{k_{1}}^{\dagger} a_{k_{2}}^{\dagger}+\ldots\right)|0\rangle|\downarrow\rangle,
\end{aligned}
$$

where the linear term $a_{k}^{\dagger}|0\rangle$ can be omitted because the expansion of the bosonic state completely reproduces the first two terms of Eq. (3). Note above that the phonon state in each level is generated by operating on the Fock state with a unitary displacement operator, called displaced Fock states . Only the first term $D\left( \pm \alpha_{k}\right)|0\rangle$ can reach the whole Hilbert-space, so no truncation is made in this sense. If the expansion is taken to the infinite order, an exact solution would be obtained. However, it is impossible to really perform an infinite order expansion. Fortunately, it will be shown later that only a few terms in the expansion would give very accurate results. In some crucial issues, converging results can actually be achieved.

As a zero-order approximation, we only consider the first term in Eq. (44). Projecting the Schrödinger equation onto the orthogonal states $\langle 0| D^{\dagger}\left(\alpha_{k}\right)$ and $\langle 0| a_{k} D^{\dagger}\left(\alpha_{k}\right)$ gives

$$
\begin{gathered}
E=\sum_{k} \omega_{k} \alpha_{k}^{2}+\sum_{k} g_{k} \alpha_{k}-\frac{\Delta}{2} \exp \left[-2 \sum_{k} \alpha_{k}^{2}\right], \\
\alpha_{k}=\frac{-\frac{1}{2} g_{k}}{\omega_{k}+\Delta \exp \left(-2 \sum_{k} \alpha_{k}^{2}\right)} .
\end{gathered}
$$

Solving $\alpha_{k}$ self-consistently we can obtain the GS energy and wavefunction immediately. It is interesting to note that the above two equations are no other than those in the previous $\mathrm{SH}$ ansatz [9]. In other words, we arrive at the previous well-known analytical results only by the zero-order approximation.

What is more, anchored to the $\mathrm{SH}$ one, the present ansatz for the wavefunction (41) can be improved by adding more displaced Fock states straightforwardly. Since the linear term is absent, we can perform the second-order correction by keeping first two terms. Note that the correlations between two bosons are fully built in. Analogously, projecting the Schrödinger equation onto $\langle 0| D^{\dagger}\left(\alpha_{k}\right),\langle 0| a_{k} D^{\dagger}\left(\alpha_{k}\right)$, and $\langle 0| a_{k_{1}} a_{k_{2}} D^{\dagger}\left(\alpha_{k}\right)$ yields the following three equations for unknown $E, \alpha_{k}$, and $b_{k_{1}, k_{2}}$,

$$
\begin{gathered}
E=\sum_{k}\left(\omega_{k} \alpha_{k}^{2}+g_{k} \alpha_{k}\right)-\frac{1}{2} \Delta \eta\left(1+4 \sum_{k} B_{k} \alpha_{k}\right) \\
\alpha_{k}=-\frac{\frac{g_{k}}{2}+2 \sum_{k^{\prime}} b_{k, k^{\prime}}\left[\left(\omega_{k^{\prime}}-\Delta \eta\right) \alpha_{k^{\prime}}+\frac{g_{k^{\prime}}}{2}\right]}{\omega_{k}+\Delta \eta\left(1+4 \sum_{k} B_{k} \alpha_{k}\right)}, \\
b_{k_{1}, k_{2}}=-\frac{B_{k_{1}} \alpha_{k_{2}}+B_{k_{2}} \alpha_{k_{1}}-\alpha_{k_{1}} \alpha_{k_{2}}\left(1+4 \sum_{k} B_{k} \alpha_{k}\right)}{2 \sum_{k} B_{k} \alpha_{k}+\left(\omega_{k_{1}}+\omega_{k_{2}}\right) /(\Delta \eta)},
\end{gathered}
$$

where

$$
B_{k}=\sum_{k^{\prime}} b_{k, k^{\prime}} \alpha_{k^{\prime}}, \eta=\exp \left[-2 \sum_{k} \alpha_{k}^{2}\right] .
$$



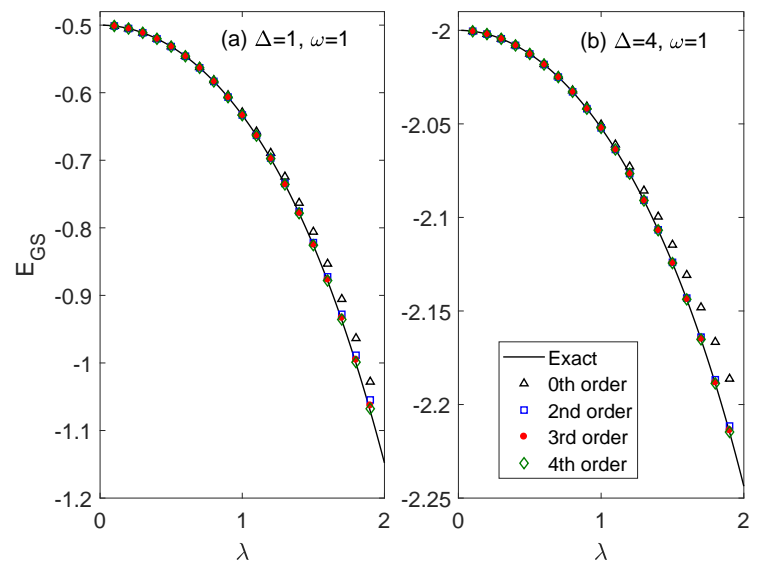

FIG. 1: (Color online) Comparisons of GS energy in the zeroth order, second-order, third-order, and fourth-order approximations with the numerically exact ones (solid lines).

$\alpha_{k}$ and $b_{k_{1}, k_{2}}$ can be obtained by solving the two coupled Eqs. (8) and (9) self-consistently, and therefore all observables can be calculated.

Note that it is a non-perturbative many-body approach. In both the zeroth- and second-order approximations, the contributions from infinite Feynman diagrams are essentially contained, as expected from the iteration spirit in the self-consistent calculations.

Proceeding as the scheme outlined above, we can straightforwardly perform the further expansion in the orthogonal displaced Fock basis $D\left(\alpha_{k}\right) \prod a_{k_{i}}^{\dagger}|0\rangle$ systematically step by step, and get the solution within any desired accuracy in principle. The challenges remain on the pathway to the high dimensional integral in the high order study, due to both the analytical derivations and exponentially increasing computational difficulties. In this paper, we are able to perform the corrections up to the fourth-order practically.

To demonstrate the effectiveness of this approach, we test it in the single-mode spin-boson model, i.e. the quantum Rabi model where the exact GS is known 32]. We present the GS energy within four different order approximations and compare with the exact ones in Fig. 1, It is interesting to note that the GS energy in high order ansatz converges rapidly to the exact ones. It follows that a finite order approximation in the present ansatz can yield sufficiently accurate results.

\section{ACCURATE RESULTS IN THE SPIN-BOSON MODEL}

Then we apply the new method to the spin-boson model. We stress here that in the present approach we do not have to discretize the bosonic energy band like in many previous studies at the very beginning. All $k$-summations involved in the derived equa-
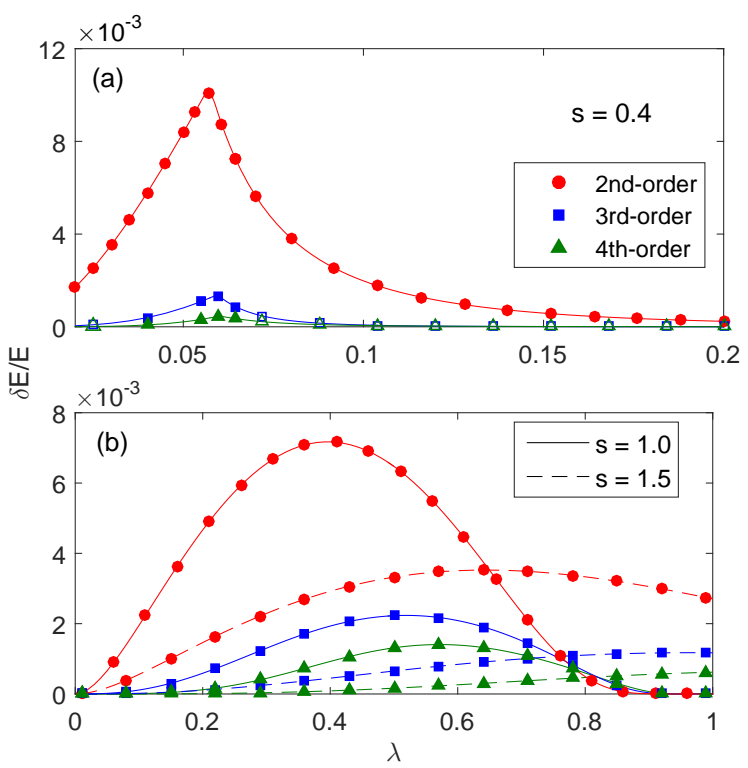

FIG. 2: (Color online) The relative difference of the ground state energy in successive ansatz $\delta E / E$ as a function of $\lambda$ in the second-, third-, and fourth-order approximations for both exponents (a) $s=0.4$ and (b) $s=1$ and 1.5.

tions can be transformed into continuous integrals like $\int_{0}^{\omega_{c}} d \omega J(\omega) I(\omega)$. It can be numerically calculated within a Gaussian-logarithmical integration with very high accuracy, as described in Appendix A in detail. Without loss of generality, we set $\Delta=0.1, \omega_{c}=1$ in the calculation if not specified.

For the sub-Ohmic baths, due to the QPT from the delocalized phase to the localized one, we should relax wavefunction (4) to the asymmetrical one straightforwardly. The zeroth-order approximation is obviously the same as the asymmetric SH ansatz [11]. The derivation up to the second-order is given in Appendix B. For the Ohmic and super-Ohmic spin-boson model, we still employ the symmetrical ansatz of the wavefunction for $\lambda<1$ where no phase transitions occur.

In Fig. 2, we present the results for the relative difference of the GS energy $\delta E / E=\left(E_{i \text { th }}-E_{(i-1) \text { th }}\right) / E_{(i-1) \text { th }}$ in the successive order for three typical bath exponents $s=0.4,1$, and 1.5 . It is found that the successive corrections decrease monotonously and tend to convergence very quickly in all cases. The relative difference for the GS energy in the fourth and third-order approximations is only around $10^{-4}$.

We also calculate the entanglement entropy between the qubit and the bath. In the spin-boson model, entanglement entropy can be obtained as [4]:

$$
S=-p_{+} \log p_{+}-p_{-} \log p_{-},
$$

where $p_{ \pm}=\frac{1}{2}\left(1 \pm \sqrt{\left\langle\sigma_{x}\right\rangle^{2}+\left\langle\sigma_{z}\right\rangle^{2}}\right)$. The entanglement entropy as a function of the coupling strength is given in Fig. 3 for $s=0.4$ and $s=1$. With successively 


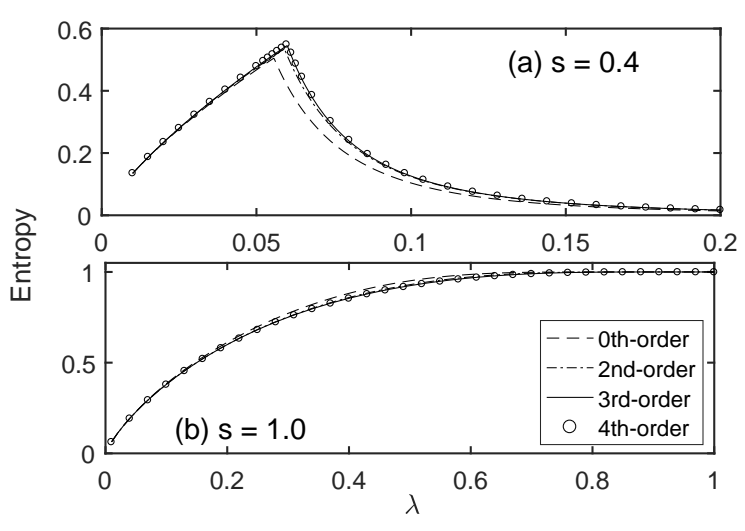

FIG. 3: (Color online) The entropy of the ground-state for both $s=0.4$ and $s=1.0$ with the zeroth, second,third and fourth order approximations.

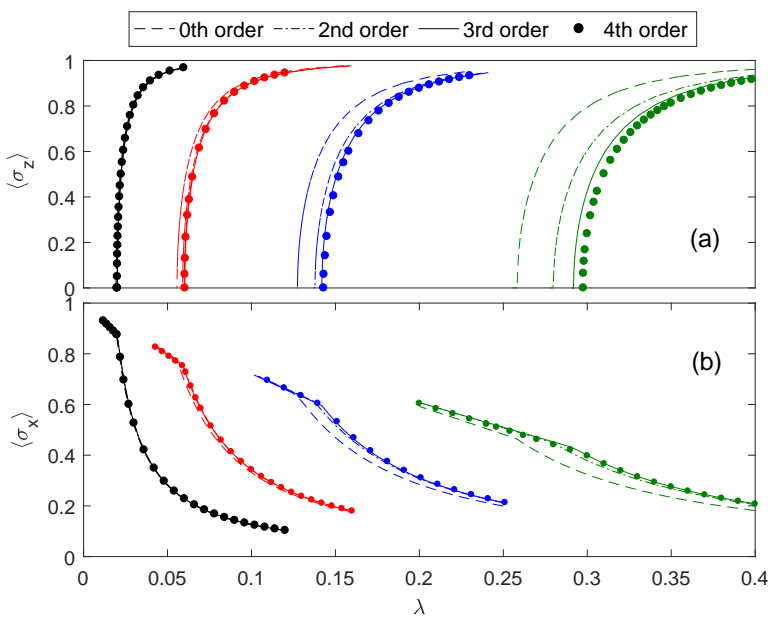

FIG. 4: (Color online) The magnetization $\left\langle\sigma_{z}\right\rangle$ (a) and $\left\langle\sigma_{x}\right\rangle$ (b) as a function of the coupling strength up to the fourthorder asymmetric SH ansatz for $s=0.2,0.4,0.6$, and 0.8 (from left to right).

higher order study, the entanglement entropy converges very quickly. The entanglement entropy exhibits a cusp for $s=0.4$, which can be used to locate the critical points of the quantum phase transition. For the Ohmic bath, the converging entropy increases monotonically with $\lambda$, and saturates at $S \approx 1$ for $\lambda>1 / 2$, in excellent agreement with previous results using the Bethe ansatz solu- tions [4].

Quantum phase transition for sub-Ohmic baths.-. For the sub-Ohmic bath, the rich physics of the quantum dissipation is second-order QPT from delocalization to localization as a consequence of the competition between the amplitude of tunneling of the spin and the effect of the dissipative bath. The magnetization $M=\left\langle\sigma_{z}\right\rangle$ can be used as an order parameter in the QPT of this model. We calculate the magnetization $M$ and evaluate the critical coupling strength $\lambda_{c}$ up to the fourth-order ansatz.

The results of magnetization for $s=0.2,0.4,0.6$, and 0.8 are exhibited in Fig. 4(a). In each order approximation, there obviously exists a critical point which separates the localized one $(M \neq 0)$ from the delocalized phase $(M=0)$. Interestingly, both $M$ and $\lambda_{c}$ display a fast converging behavior. Especially for bath exponents $s=0.2$ and $s=0.4$, the converging magnetization has been already achieved. We believe that the almost exact GS results in the bath exponent regime $0<s<1 / 2$ can be obtained in the present approach up to the fourthorder ansatz.

However, for $s>1 / 2$, the convergence is not arrived at until the fourth-order calculations. It is expected that the more accurate results could be obtained in the further corrections, which is however prohibitively expensive in the high dimensional continuous integral. As found recently by Blunden-Codd et al., 13], the quantum criticality associated to an interacting fixed point 33 in this nontrivial regime can be only touched by the very accurate wavefunction where with at least a hundred of singlemode coherent states are required, which is beyond the scope of the present study.

The renormalized tunneling matrix element $\left\langle\sigma_{x}\right\rangle$ in the GS for different value of $s$ is also presented in Fig. 4(b). A fast converging behavior is also found. Note that, after the critical point, it decreases more rapidly, but does not vanish.

Both the magnetization and entanglement entropy can give the same $\lambda_{c}$. For convenience, we list critical coupling strengths by the present different order approximations in Table I. The corresponding QMC results, which are drawn from Ref. [21], are also collected for comparison. $\lambda_{c}$ generally increases with the increasing order. Convergency is achieved obviously for $s<1 / 2$. Interestingly, $\lambda_{c}=0.0600$ for $s=0.4$ in our fourth-order study is almost identical to the previous QMC one 0.0601 [21].

TABLE I: The critical coupling strengths within different order approximations are collected. The sixth column presents those by quantum Monte Carlo simulations [21].

\begin{tabular}{llllll}
\hline \hline$s$ & $0 t h$ & $2 n d$ & $3 r d$ & $4 t h$ & $Q M C^{\text {Ref.[21] }}$ \\
\hline 0.2 & 0.0195 & 0.02005 & 0.02013 & 0.02014 & 0.0175 \\
0.4 & 0.0557 & 0.0590 & 0.0599 & 0.0600 & 0.0601 \\
0.6 & 0.127 & 0.138 & 0.142 & 0.143 & 0.155 \\
0.8 & 0.258 & 0.280 & 0.292 & 0.297 & 0.359 \\
\hline \hline
\end{tabular}




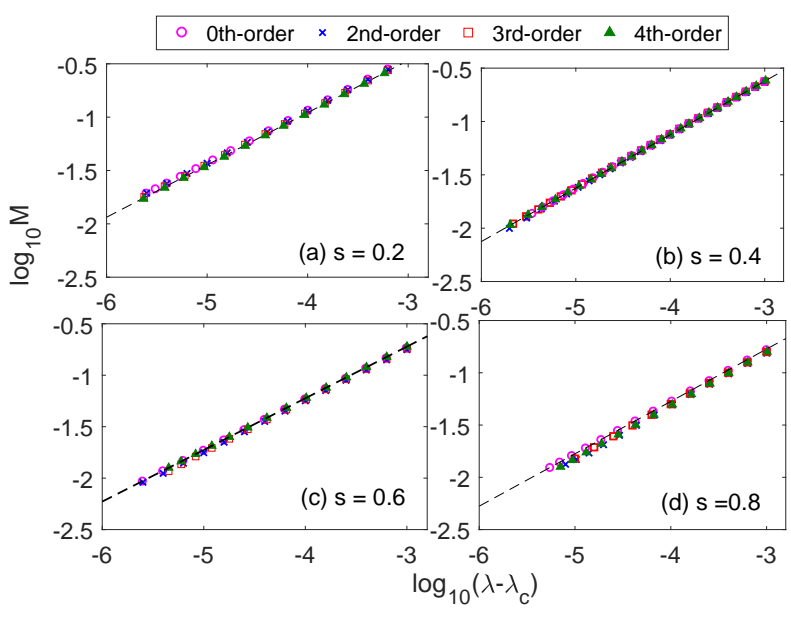

FIG. 5: (Color online) The log-log plot of the magnetization $M$ as a function of $\lambda-\lambda_{c}$ for $s=0.2,0.4,0.6$, and 0.8. The power law curves with $\beta=0.5$ are denoted by the dashed line.

Discretization of the energy spectrum of the continuum bath should be performed at the very beginning in many advanced numerical approaches [17 20, 22 26, 34], except in the QMC where the bath is integrated out. The artificial discretization definitely yields quantitative calculation errors. Only QMC simulations may in principle provide reliable and unique critical points with high accuracy. One may note that our result for $\lambda_{c}$ for $s=0.2$ is close to but a little bit larger than the QMC one. It unlikely converges to the QMC value due to the trend of convergence. The statistical error in QMC simulations may account for this slight difference.

In the second-order QPT, the order parameter should display a power law behavior near the critical point $M \propto\left(\lambda-\lambda_{c}\right)^{\beta}$. We present the magnetization within the second-, third-order and fourth-order ansatz as a function of $\lambda-\lambda_{c}$ in a log-log plot for $s=0.2,0.4,0.6$, and 0.8 in Fig. [5] It is observed that the curves almost coincide in the whole coupling regime. A very nice power-law behavior over two decades with an exponent $\beta=0.5 \pm 0.01$ is demonstrated for all cases. Therefore even the fourthorder study does not modify the exponent $\beta$ either, indicating a robust mean-field nature in the regime $s<1 / 2$ of this model.

Biased spin-boson model.- Extension to the biased spin-boson model can be performed straightforwardly. In this case, a static bias term $\frac{\epsilon}{2} \sigma_{z}$ is added to Hamiltonian (11). Obviously, the asymmetrical wavefunction should be entailed in the asymmetrical spin-boson model with any baths. Nazir et al. found that the GS magnetization $(M)$ within the $\mathrm{SH}$ ansatz jumps to -1 , i.e. the value for the fully localized state, for some value of $\lambda$ [35], whereas many advanced approaches have explicitly led

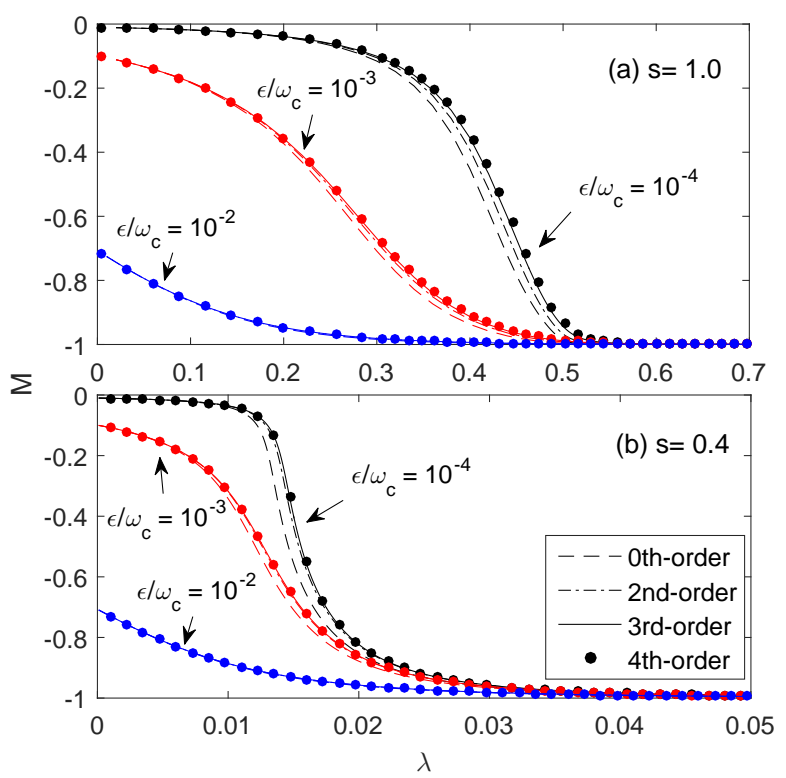

FIG. 6: (Color online) The log-log plot of the magnetization $M$ as a function of $\lambda-\lambda_{c}$ for (a) $s=1.0$, and (b) $s=0.4$. The power law curves with $\beta=0.5$ are denoted by the dashed line.

to the smooth crossover behavior.

We set the same parameters as in Ref. [35], $\Delta / \omega_{c}=$ 0.01 , and also calculate $M$ as a function of $\lambda$ within the present ansatz. As shown in Fig. 6 that, for the Ohmic baths, the unphysical discontinuous "jump" for small bias $\epsilon$ does not appear in any order approximations. We believe that the previous surprising jump is caused by the symmetrical $\mathrm{SH}$ variational wavefunction used in Ref. [35]. Moreover, fast convergence of the magnetization is shown in Fig. 6 for both $s=1$ and 0.4 . Especially, for $s=0.4$, a converging result is arrived even in the third-order ansatz. It is demonstrated that the present approach is also quite efficient in the biased case.

\section{CONCLUSION}

Previous well-known SH polaron ansatz in the spinboson model is improved successively in this paper. The GS wavefunction can be written in the expansion of orthogonal displaced Fock states. Projecting the Schrödinger equation onto orthogonal displaced Fock states with increasing order gives a set of coupled equations. Solving the equations self-consistently allows for the GS observables. The zeroth-order one is just the same as the SH ansatz. More importantly, it can be easily extended to the high order, which is actually the expansion 
anchored around SH ansatz. More accurate results can then be achieved with increasing order where correlations among more bosons are included. In principle, the exact results could be archived in the infinite-order study, which is, however, very challenging at the moment. Fortunately, very accurate results can be obtained in a few higher order calculations that we can performed practically. Especially in the sub-Ohmic spin-boson model, the fourth-order ansatz can yield the converging results for $0<s<1 / 2$. The present approach is also suited to the biased spin-Boson model. It is expected that this approach paves the way to the true exact solution analytically. Generalizations to quantum dynamics at both zero and finite temperatures are progressing.

\section{Appendix A: Gaussian-logarithmical integration for the continuous integral}

Here we demonstrate how to transform the $k$-summation appearing in Eqs. (5-10) into the continuous integral and then illustrate an effective numerical calculation with high accuracy.

In the zero-order approximation, also the well known SH ansatz, we can set

$$
\alpha_{k}=\alpha_{k}^{\prime} g_{k}
$$

Eq. (6) then becomes

$$
\alpha_{k}^{\prime}=-\frac{1 / 2}{\omega_{k}+\Delta \exp \left(-2 \sum_{k} \alpha_{k}^{\prime 2} g_{k}^{2}\right)},
$$

so $\alpha_{k}^{\prime}$ is only related to $g_{k}$ implicitly.

In the second-order approximation, we can set

$$
\begin{aligned}
\alpha_{k} & =\alpha_{k}^{\prime} g_{k}, \\
b_{k_{1}, k_{2}} & =b_{k_{1}, k_{2}}^{\prime} g_{k_{1}} g_{k_{2}} .
\end{aligned}
$$

Inserting to Eqs. (8) and (9) gives

$$
\alpha_{k}^{\prime}=\frac{-\frac{1}{2}+2 \sum_{k^{\prime}} g_{k^{\prime}}^{2} b_{k, k^{\prime}}^{\prime}\left[\left(\omega_{k^{\prime}}-\Delta \eta\right) \alpha_{k^{\prime}}^{\prime}+1 / 2\right]}{\omega_{k}+\Delta \eta(1+4 \zeta)},
$$

$$
b_{k_{1}, k_{2}}^{\prime}=\frac{\alpha_{k_{1}}^{\prime} \alpha_{k_{2}}^{\prime}(1+4 \zeta)-\sum_{k^{\prime}} g_{k^{\prime}}^{2} \alpha_{k^{\prime}}^{\prime}\left(b_{k_{1}, k^{\prime}}^{\prime} \alpha_{k_{2}}^{\prime}+b_{k_{2}, k^{\prime}}^{\prime} \alpha_{k_{1}}^{\prime}\right)}{2 \zeta+\left(\omega_{k_{1}}+\omega_{k_{2}}\right) /(\Delta \eta)}
$$

where

$$
\zeta=\sum_{k} g_{k}^{2} \sum_{k^{\prime}} g_{k^{\prime}}^{2} b_{k, k^{\prime}}^{\prime} \alpha_{k^{\prime}}^{\prime} \alpha_{k}^{\prime} .
$$

Given $g_{k}$, both $\alpha_{k}^{\prime}$ and $b_{k_{1}, k_{2}}^{\prime}$ can be obtained selfconsistently. Note that each $k$-summation takes the form of $\sum_{k} g_{k}^{2} I(k)$ where $I(k)$ does not depend on $g_{k}^{2}$ explicitly, and so both $\alpha_{k}^{\prime}$ and $b_{k_{1}, k_{2}}^{\prime}$ are functionals of $g_{k}$. Without loss of generality, $k$ is corresponding to $\omega$ one by one, the $k$-summation can be transformed to the $\omega$ integral as

$$
\sum_{k} g_{k}^{2} I(k) \rightarrow \int_{0}^{\omega_{c}} d \omega \frac{J(\omega)}{\pi} I(\omega),
$$

so we have

$$
\begin{gathered}
\alpha^{\prime}(\omega)=\frac{-\frac{1}{2}+\xi(\omega)-2 \Delta \eta \chi(\omega)}{\omega+\Delta \eta(1+4 \zeta)}, \\
b^{\prime}\left(\omega_{1}, \omega_{2}\right)=\frac{\alpha^{\prime}\left(\omega_{1}\right) \alpha^{\prime}\left(\omega_{2}\right)(1+4 \zeta)-\kappa\left(\omega_{1}, \omega_{2}\right)}{2 \zeta+\left(\omega_{1}+\omega_{2}\right) /(\Delta \eta)},
\end{gathered}
$$

where

$$
\begin{aligned}
\xi(\omega) & =\int_{0}^{\omega_{c}} d \omega^{\prime} \frac{J\left(\omega^{\prime}\right)}{\pi}\left[2 \omega^{\prime} \alpha^{\prime}\left(\omega^{\prime}\right)+1\right] b^{\prime}\left(\omega, \omega^{\prime}\right), \\
\chi(\omega) & =\int_{0}^{\omega_{c}} d \omega^{\prime} \frac{J\left(\omega^{\prime}\right)}{\pi} \alpha^{\prime}\left(\omega^{\prime}\right) b^{\prime}\left(\omega, \omega^{\prime}\right), \\
\kappa\left(\omega_{1}, \omega_{2}\right) & =\chi\left(\omega_{1}\right) \alpha^{\prime}\left(\omega_{2}\right)+\chi\left(\omega_{2}\right) \alpha^{\prime}\left(\omega_{1}\right),
\end{aligned}
$$

are some functions of $\omega$, and

$$
\begin{aligned}
& \zeta=\int_{0}^{\omega_{c}} d \omega \frac{J(\omega)}{\pi} \int_{0}^{\omega_{c}} d \omega^{\prime} \frac{J\left(\omega^{\prime}\right)}{\pi} \alpha^{\prime}(\omega) \alpha^{\prime}\left(\omega^{\prime}\right) b^{\prime}\left(\omega, \omega^{\prime}\right), \\
& \eta=\exp \left[-2 \int_{0}^{\omega_{c}} d \omega^{\prime} \frac{J\left(\omega^{\prime}\right)}{\pi} \alpha^{\prime 2}\left(\omega^{\prime}\right)\right],
\end{aligned}
$$

are constants.

The GS energy (7) can be expressed as

$$
E=\int_{0}^{\omega_{c}} d \omega \frac{J(\omega)}{\pi} \alpha^{\prime}(\omega)\left[\omega \alpha^{\prime}(\omega)+1\right]-\frac{1}{2} \Delta \eta(1+4 \zeta) .
$$

The self-consistent solutions of two coupled equations Eqs. (A1) and (A2) are in no way obtained analytically, numerical calculations should be performed. Note that the low frequency modes play the dominant role in the QPT of the sub-ohmic spin-boson model. There is an infrared divergence of the integrand like $\int_{0}^{\omega_{c}} \omega^{s-2} d \omega$ in the limit of $\omega \rightarrow 0$ for the sub-Ohmic and Ohmic baths, which is called as the infrared catastrophe. Thanks to the Gaussian quadrature rules, where the zero frequency is not touched. We can discretize the whole frequency interval with Gaussian grids, the integral can be numerically exactly achieved with a large number of Gaussian grids. It is very time consuming to calculate the integral in this way, especially for high dimensional integral involved in the high order approximation. According to the struc- 
ture of the integrand, it is not economical to deal with the high and low frequency regime on the equal footing.

To increase the efficiency, we combine the logarithmic discretization with Gaussian quadrature rule [36]. First, we divide the $\omega$ interval $[0,1]$ into $L+1$ sub-intervals as $\left[\Lambda^{-(l+1)}, \Lambda^{-l}\right](l=0,1,2, L-1)$ and $\left[0, \Lambda^{-L}\right]$, then we apply the Gaussian quadrature rule to each logarithmical sub-interval. So the continuous integral is calculated by the following summation

$$
\int_{0}^{1} J(\omega) I(\omega) d \omega=\sum_{l=0}^{L} \sum_{n=1}^{N} W_{l, n} J\left(\omega_{l, n}\right) I\left(\omega_{l, n}\right),
$$

where $N$ is the number of gaussian points inserted in each sub-interval, $W_{l, n}$ is corresponding Gaussian weight. After careful examination, we find that the Gaussianlogarithmical integration for integrand in this problem converges if set $L=6, N=9$, and $\Lambda=9$, which are used in this work.

\section{Appendix B: Formalism for sub-Ohmic baths}

In the sub-Ohmic spin-boson model, the GS wavefunction can be generally expressed in the orthogonal displaced Fock basis $D\left(\alpha_{k}\right) \prod a_{k_{i}}^{\dagger}|0\rangle$ as

$$
\begin{aligned}
& |\Psi\rangle=D\left(\alpha_{k}\right)\left(1+\sum_{k_{1}, k_{2}} b_{1}\left(k_{1}, k_{2}\right) a_{k_{1}}^{\dagger} a_{k_{2}}^{\dagger}+\sum_{k_{1}, k_{2}, k_{3}} c_{1}\left(k_{1}, k_{2}, k_{3}\right) a_{k_{1}}^{\dagger} a_{k_{2}}^{\dagger} a_{k_{3}}^{\dagger}+\ldots\right)|0\rangle|\uparrow\rangle \\
& +D\left(\beta_{k}\right)\left(r+\sum_{k_{1}, k_{2}} b_{2}\left(k_{1}, k_{2}\right) a_{k_{1}}^{\dagger} a_{k_{2}}^{\dagger}+\sum_{k_{1}, k_{2}, k_{3}} c_{2}\left(k_{1}, k_{2}, k_{3}\right) a_{k_{1}}^{\dagger} a_{k_{2}}^{\dagger} a_{k_{3}}^{\dagger}+\ldots\right)|0\rangle|\downarrow\rangle,
\end{aligned}
$$

where $r, \alpha_{k}, \beta_{k}$, and $b_{i}, c_{i}(i=1,2)$ are asymmetrical coefficients to be determined.

In the zero-order approximation, we only select the first term in Eq. (B1). Projecting the Schrödinger equation in the upper level onto the orthogonal basis $\langle 0| D^{\dagger}\left(\alpha_{k}\right)$ and $\langle 0| a_{k} D^{\dagger}\left(\alpha_{k}\right)$ and in the lower level onto $\langle 0| D^{\dagger}\left(\beta_{k}\right)$ and $\langle 0| a_{k} D^{\dagger}\left(\beta_{k}\right)$ results in

$$
\begin{aligned}
\sum_{k}\left(\omega_{k} \alpha_{k}^{2}+g_{k} \alpha_{k}\right)-\frac{\Delta}{2} r \Gamma & =E, \\
\omega_{k} \alpha_{k}+\frac{1}{2} g_{k}+\frac{\Delta}{2} r \Gamma D_{k} & =0,
\end{aligned}
$$

and

$$
\begin{aligned}
\sum_{k}\left(\omega_{k} \beta_{k}^{2}-g_{k} \beta_{k}\right)-\frac{\Delta}{2 r} \Gamma & =E, \\
\omega_{k} \beta_{k}-\frac{1}{2} g_{k}-\frac{\Delta}{2 r} \Gamma D_{k} & =0
\end{aligned}
$$

where

$$
\begin{aligned}
\Gamma & =\exp \left[-\frac{1}{2} \sum_{k} D_{k}^{2}\right], \\
D_{k} & =\alpha_{k}-\beta_{k},
\end{aligned}
$$

which are the same as those obtained variationally within the generalized SH ansatz [11].

In the second-order approximation, the first two terms in each level of Eq. (B1) are kept. Proceeding as procedures outlines above, projecting the Schrödinger equation in the upper level onto the orthogonal states $\langle 0| D^{\dagger}\left(\alpha_{k}\right),\langle 0| a_{k} D^{\dagger}\left(\alpha_{k}\right)$, and $\langle 0| a_{k_{1}} a_{k_{2}} D^{\dagger}\left(\alpha_{k}\right)$ and in the lower level onto $\langle 0| D^{\dagger}\left(\beta_{k}\right),\langle 0| a_{k} D^{\dagger}\left(\beta_{k}\right)$, and $\langle 0| a_{k_{1}} a_{k_{2}} D^{\dagger}\left(\beta_{k}\right)$ yield the following six equations

$$
\begin{gathered}
\sum_{k}\left[\omega_{k} \alpha_{k}^{2}+g_{k} \alpha_{k}\right]-\frac{\Delta}{2} \Gamma\left[r+\sum_{k} B_{k} D_{k}\right]=E \\
r \sum_{k}\left[\omega_{k} \beta_{k}^{2}-g_{k} \beta_{k}\right]-\frac{\Delta}{2} \Gamma\left[1+\sum_{k} A_{k} D_{k}\right]=r E \\
{\left[\omega_{k} \alpha_{k}+\frac{g_{k}}{2}\right]+\sum_{k^{\prime}} 2 b_{1}\left(k, k^{\prime}\right)\left[\omega_{k^{\prime}} \alpha_{k^{\prime}}+\frac{g_{k^{\prime}}}{2}\right]-\Delta \Gamma B_{k}+\frac{\Delta}{2} \Gamma D_{k}\left[r+\sum_{k} B_{k} D_{k}\right]=0,}
\end{gathered}
$$




$$
\begin{gathered}
r\left[\omega_{k} \beta_{k}-\frac{g_{k}}{2}\right]+\sum_{k^{\prime}} 2 b_{2}\left(k, k^{\prime}\right)\left[\omega_{k^{\prime}} \beta_{k^{\prime}}-\frac{g_{k^{\prime}}}{2}\right]+\Delta \Gamma A_{k}-\frac{\Delta}{2} \Gamma D_{k}\left[1+\sum_{k} A_{k} D_{k}\right]=0, \\
b_{1}\left(k_{1}, k_{2}\right)\left(\omega_{k_{1}}+\omega_{k_{2}}\right)+\frac{\Delta}{2} \Gamma\left[r+\sum_{k} B_{k} D_{k}\right] b_{1}\left(k_{1}, k_{2}\right) \\
-\frac{\Delta}{2} b_{2}\left(k_{1}, k_{2}\right) \Gamma+\frac{\Delta}{2} \Gamma\left[B_{k_{1}} D_{k_{2}}+B_{k_{2}} D_{k_{1}}\right]-\frac{\Delta}{4} \Gamma D_{k_{1}} D_{k_{2}}\left[r+\sum_{k} B_{k} D_{k}\right]=0, \\
b_{2}\left(k_{1}, k_{2}\right)\left(\omega_{k_{1}}+\omega_{k_{2}}\right)+\frac{\Delta}{2 r} \Gamma\left[1+\sum_{k} A_{k} D_{k}\right] b_{2}\left(k_{1}, k_{2}\right) \\
-\frac{\Delta}{2} b_{1}\left(k_{1}, k_{2}\right) \Gamma+\frac{\Delta}{2} \Gamma\left[A_{k_{1}} D_{k_{2}}+A_{k_{2}} D_{k_{1}}\right]-\frac{\Delta}{4} \Gamma D_{k_{1}} D_{k_{2}}\left[1+\sum_{k} A_{k} D_{k}\right]=0,
\end{gathered}
$$

where

$$
\begin{aligned}
& A_{k}=\sum_{k^{\prime}} b_{1}\left(k^{\prime}, k\right) D_{k^{\prime}} \\
& B_{k}=\sum_{k^{\prime}} b_{2}\left(k^{\prime}, k\right) D_{k^{\prime}} .
\end{aligned}
$$

The self-consistent solutions for the above coupled equations will give all results in the second-order study. If set $r=1, \alpha_{k}=-\beta_{k}$ and $b_{1}\left(k_{1}, k_{2}\right)=b_{2}\left(k_{1}, k_{2}\right)$, Eqs. (8) and (9) for the symmetric case are recovered completely.
It is straightforward to perform the third-order and fourth-order studies by further adding the third and the fourth terms in Eq. (B1). The derivations are lengthy and not shown here.

ACKNOWLEDGEMENTS This paper is supported by the National Science Foundation of China (No. 11474256 and No. 11674285), and the National Key Research and Development Program of China (No. 2017YFA0303002).

* Corresponding author. Email:qhchen@zju.edu.cn
[1] A. J. Leggett et al., Rev. Mod. Phys. 59, 1(1987).

[2] U. Weiss, Quantum Dissipative Systems (World Scientific, Singapore, 1993).

[3] K. L. Hur, P. D. Beaupré, and W. Hofstetter, Phys. Rev. Lett. 99, 126801(2007).

[4] A. Kopp and K. Le Hur, Phys. Rev. Lett. 98, 220401 (2007)

[5] M. Thorwart and P. Hanggi, Phys. Rev. A 65, 012309 (2001); T. A. Costi and R. H. McKenzie, Phys. Rev. A 68, 034301 (2003).

[6] Y. Makhlin, G. Schon, and A. Shnirman, Phys. Mod. Phys. 73, 357(2001).

[7] T. Renger and R. A. Marcus, J. Chem. Phys. 116, 9997(2002).

[8] A. Omerzu, M. Licer, T. Mertelj, V. V. Kabanov, and D. Mihailovic, Phys. Rev. Lett. 93, 218101(2004).

[9] R. Silbey, and R. A. Harris, J. Chem. Phys. 80, 2615 (1984).

[10] Z. G. Lu and H. Zheng, Phys. Rev. B. 75, 054302(2007); Q. Wang, A. Y. Hu, and H. Zheng, Phys. Rev. B. 80, 214301(2009); Q.-J. Tong, J.-H. An, H.-G. Luo, and C. H. Oh, Phys. Rev. B 84, 174301 (2011).

[11] A. W. Chin, J. Prior, S. F. Huelga and M. B. Plenio, Phys. Rev. Lett. 107, 160601 (2011).

[12] S. Bera, S. Florens, H. U. Baranger, N. Roch, A. Nazir,
A. W. Chin, Phys. Rev. B 89, 121108(R) (2014); S. Bera, A. Nazir, A. W. Chin, H. U. Baranger, S. Florens, Phys. Rev. B 90, 075110 (2014).

[13] Z. Blunden-Codd, S. Bera, B. Bruognolo, N.-O. Linden, A. W. Chin, J. von Delft, A. Nazir, and S. Florens, Phys. Rev. B 95, 085104 (2017).

[14] H. Zheng and Z. G. Lu, J. Chem. Phys. 138, 174117 (2013).

[15] C. K. Lee, J. M. Moix, and J. Cao, J. Chem. Phys. 136, 204120 (2012); C. K. Lee, J. Cao, and J. B. Gong Phys. Rev. E 86, 021109 (2012).

[16] C. R. Duan, Z. F. Tang, J. S. Cao, and J. L. Wu, Phys. Rev. B 95, 214308 (2017); C. R. Duan, Q. L. Wang, Z. F. Tang, and J. L. Wu, J. Chem. Phys. 147, 164112 (2017).

[17] R. Bulla, N. H. Tong, and M. Vojta, Phys. Rev. Lett. 91, 170601(2003); M. Vojta, N. H. Tong, and R. Bulla, Phys. Rev. Lett.94, 070604 (2005); R. Bulla, H. J. Lee, N. H. Tong, and M. Vojta, Phys. Rev. B. 71, 045122(2005).

[18] M. Vojta, N. H. Tong, and R. Bulla, Phys. Rev. Lett.102, 249904(E) (2009); M. Vojta, R. Bulla, F. Guttge, and F. Anders, Phys. Rev. B 81, 075122 (2010).

[19] N. H. Tong and Y. H. Hou, Eur. Phys. J. B 78, 127 (2010); Phys. Rev. B 85, 144425(2012).

[20] For a review see: M. Vojta, Philos. Mag. 86, 1807(2006).

[21] A. Winter, H. Rieger, M. Vojta, and R. Bulla, Phys. Rev. 
Lett. 102, 030601 (2009).

[22] A. Alvermann and H. Fehske, Phys. Rev. Lett. 102, 150601 (2009).

[23] Y. Y. Zhang, Q. H. Chen and K. L. Wang, Phys. Rev. B 81, 121105(R) (2010).

[24] H. Wong, and Z. D. Chen, Phys. Rev. B. 77, 174305(2008).

[25] C. Guo, A. Weichselbaum, J. V. Delft, and M. Vojta, Phys. Rev. Lett. 108, 160401 (2012).

[26] M. F. Frenzel and M. B. Plenio, New Journal of Physics 15, 073046(2013).

[27] M. L. Wall, A. Safavi-Naini, and A. M. Rey, Phys. Rev. A 94, 053637 (2016).

[28] F. A. Y. N. Schröder and A. W. Chin, Phys. Rev. B 93, 075105 (2016).

[29] J. Haegeman, C. Lubich, I. Oseledets, B. Vandereycken, and F. Verstraete, Phys. Rev. B 94, 165116 (2016).

[30] Q. H. Chen, T. Liu, Y. Y. Zhang, and K. L. Wang, Europhys. Lett. 96, 14003 (2011).

[31] D. Braak, Phys. Rev. Lett. 107, 100401 (2011).

[32] Q. H Chen, C. Wang, S. He, T. Liu, and K. L. Wang, Phys. Rev. A 86, 023822(2012).

[33] M. Vojta, Phys. Rev. B. 85, 115113(2012)

[34] S. Kirchner, Q. Si, and K. Ingersent, Phys. Rev. Lett. 102, 166405 (2009); S. Kirchner, K. Ingersent, and Q. Si, Phys. Rev. B. 85, 075113(2012).

[35] A. Nazir, D. P. S. McCutcheon, and A. W. Chin, Phys. Rev. B. 85, 224301(2012).

[36] R. L. Burden and J. D. Faires, Numerical Analysis, 9th ed. (Brooks/Cole, Cengage Learning productors, Nelsion Education, Ltd, Boston, 2011), p. 228. 